\title{
Solving the cooling flow problem of galaxy clusters by dark matter neutralino annihilation
}

$\operatorname{AUTHOR}(S)$ :

Totani, $T$

CITATION:

Totani, T. Solving the cooling flow problem of galaxy clusters by dark matter neutralino annihilation. PHYSICAL REVIEW LETTERS 2004, 92(19): 191301.

ISSUE DATE:

2004-05-14

URL:

http://hdl.handle.net/2433/49882

RIGHT:

Copyright 2004 American Physical Society 


\title{
Solving the Cooling Flow Problem of Galaxy Clusters by Dark Matter Neutralino Annihilation
}

\author{
Tomonori Totani \\ Department of Astronomy, Kyoto University, Sakyo-ku, Kyoto, 606-8502, Japan
}

(Received 3 January 2004; published 14 May 2004)

\begin{abstract}
Recent X-ray observations revealed that strong cooling flow of intracluster gas is not present in galaxy clusters, even though it is predicted theoretically if there is no additional heating source. I show that relativistic particles produced by dark matter neutralino annihilation in cluster cores provide a sufficient heating source to suppress the cooling flow, under reasonable astrophysical circumstances including adiabatic growth of central density profile, with appropriate particle physics parameters for dark matter neutralinos. In contrast to other astrophysical heat sources, such as active galactic nuclei, this process is a steady and stable feedback over cosmological time scales after turned on.
\end{abstract}

Diffuse thermal $x$-ray emission by bremsstrahlung of intracluster gas at a temperature of $\sim 10 \mathrm{keV}$ has been observed from galaxy clusters for many decades. More than half of the clusters are called cooling flow (CF) clusters, since cooling time of central cores is less than the Hubble time, and theorists predicted the existence of strong CF in such systems with a rate of $\gtrsim 100 M_{\odot} \mathrm{yr}^{-1}$. However, recent $\mathrm{x}$-ray observations failed to reveal evidence of $\mathrm{CFs}$, requiring that, somewhat ironically, there must be some heat source to suppress CFs in "cooling flow clusters" [1]. The required amount of heating is $\sim 10^{45} \mathrm{ergs} / \mathrm{s}$ over a time scale of the cluster age $\left(\sim 10^{10} \mathrm{yr}\right)$.

Thermal conduction is probably playing a role, especially by preventing the gas from becoming thermally unstable, but a fine-tuning is necessary, and thermal conduction alone does not successfully explain all clusters [2]. Another heating source popularly discussed is active galactic nuclei (AGNs) [3], but efficiency must be very high ( $210 \%$ of the black hole rest mass energy) [4]. Generally AGNs have intermittent activity, and the accretion rate is likely determined by the dynamics of a small region around the supermassive black holes (SMBHs). Hence, it might be somewhat surprising if all clusters are kept stable over the $\gtrsim 100 \mathrm{kpc}$ scale by feedback of central AGNs.

Clusters are gravitationally dominated by the cold dark matter, for which the leading candidate is the lightest supersymmetric (SUSY) particles, plausibly the neutralino $\chi$. The neutralino mass is limited in the range $30 \mathrm{GeV} \lesssim m_{\chi} \lesssim 10 \mathrm{TeV}$, and the thermally averaged annihilation cross section is related to the relic density as $\langle\sigma v\rangle \sim 3 \times 10^{-27} /\left(\Omega_{\chi} h^{2}\right) \mathrm{cm}^{-3} \mathrm{~s}^{-1}$ (see, e.g., [5] for a review). Detectability of annihilation products from high density regions such as the galactic center (GC) has been widely discussed ([5,6], and references therein). Here I consider a possibility that annihilation products may contribute to the heating of intracluster gas. Exotic particle dark matter interacting with baryons has been proposed to solve the CF problem [7], but our scenario is based on theoretically better-motivated neutralino dark matter. Neutralino annihilation in galaxy clusters has been considered by Ref. [8] to explain diffuse radio halos observed in some clusters. The correlation of radio halos with merging clusters [9], however, indicates that such halos are formed by cosmic-ray electrons produced by merger shocks. The change of central density profile by SMBH was not taken into account in Ref. [8], without which the annihilation luminosity is too small to solve the CF problem.

Since we are interested in the relatively central region of a cluster, I use the following form of the dark matter density profile at $r \leqq r_{0}: \rho=\rho_{0}\left(r / r_{0}\right)^{-\gamma}$ with $r_{0}=$ $0.5 \mathrm{Mpc}$ and $\rho_{0}=10^{-25} \mathrm{~g} \mathrm{~cm}^{-3}$, for a typical rich cluster of $M_{15} \equiv M_{\mathrm{cl}} /\left(10^{15} M_{\odot}\right) \sim 1$. In the latest numerical simulations of cosmological structure formation, the density profile around the center generally becomes cusps with $\gamma \sim 1-1.5$ [10]. Here I use $\gamma=1$, since $\gamma=1.5$ is not supported by a recent $\mathrm{x}$-ray observation [11]. It is easy to see that neutralino annihilation has only negligible effects on cluster energetics, when simply this density profile is applied. The contribution from the cusp to annihilation rate $\left(\propto \rho^{2}\right)$ is convergent with $r \rightarrow 0$ and unimportant when $\gamma<1.5$. Therefore it is a good approximation to estimate the total annihilation luminosity, $L_{\chi \chi}$, using a mean cluster density, $\rho_{0}$. It becomes $L_{\chi \chi} \sim 2\langle\sigma v\rangle \rho_{0} M_{\mathrm{cl}} c^{2} / m_{\chi} \sim 2 \times 10^{40}\langle\sigma v\rangle_{-26} \times$ $m_{2}^{-1} M_{15} \mathrm{ergs} / \mathrm{s}$, where $m_{2}=m_{\chi} /(100 \mathrm{GeV})$ and $\langle\sigma v\rangle_{-26}=\langle\sigma v\rangle /\left(10^{-26} \mathrm{~cm}^{3} \mathrm{~s}^{-1}\right)$. This is about 5 orders of magnitude lower than that required to suppress the CF.

However, the situation drastically changes if there is a density "spike" associated with a central SMBH. Adiabatic growth of a SMBH at the center of a preexisting halo produces a spike in the density profile within $r \lesssim r_{s}$ from the center, which is even steeper than original cusps [12]. The power-law index of the spike density profile, $\gamma_{s}$, is related to that of the original cusp as $\gamma_{s}=(9-2 \gamma) /$ $(4-\gamma)=2.33-2.4$ for $\gamma=1-1.5$. The spike radius $r_{s}$ is a radius within which the enclosed mass of the halo is the same as the central SMBH. Since $\gamma_{s}>1.5$, the volume 
integration of the annihilation rate is divergent with $r \rightarrow 0$, and hence a huge enhancement of $L_{\chi \chi}$ is possible from the very central part of the halo.

If this enhancement is happening in the center of our Galaxy, a much stronger flux of annihilation gamma rays and cosmic rays is expected than previously thought, and already existing experimental/observational limits exclude a considerable SUSY parameter space [12]. However, several authors have argued that such a process is rather unlikely to occur in the GC $[13,14]$. The initial SMBH mass before adiabatic growth should be much smaller than the final one, and it must be placed to the dynamical center of the halo, but the dynamical friction time for that may be larger than the Hubble time. The mass density of the GC is dominated by baryons rather than by dark matter, and violent processes such as star formation and supernova explosions might disrupt cold orbits of dark matter particles required for the spike formation. Mergers between halos containing SMBHs may lead to a flatter density profile than original cusps.

However, the center of galaxy clusters, especially those having cooling cores within the Hubble time, appear to be the best site for the spike growth to happen. All CF clusters seem to be dynamically well evolved systems, and a single giant $\mathrm{cD}$ galaxy is placed on the gravitational center where the $\mathrm{x}$-ray surface brightness peaks [15]. Recent Chandra observations (e.g., [11]) have shown that the dark matter dominates the mass density with a profile consistent with $\gamma=1$ down to $\sim \mathrm{kpc}$, and probably further down to the SMBH scale. If strong CF with the theoretically predicted accretion rate $\left(\sim 10^{2} M_{\odot} \mathrm{yr}^{-1}\right)$ occurred at some epoch in early cluster evolution and this accretion is used to feed the SMBH of a cD galaxy, the SMBH mass could grow to $M_{\bullet, 10} \equiv M_{\bullet} /\left(10^{10} \mathrm{M}_{\odot}\right) \sim 1$ within $\gtrsim 10^{8} \mathrm{yr}$ depending on the efficiency of mass accretion onto the SMBH. This is much shorter than the typical cluster age, while the orbital period at the spike radius $r_{s}=1.5 M_{\bullet, 10}^{1 / 2} \mathrm{kpc}$ is $5.7 \times 10^{7} M_{\bullet, 10}^{1 / 4} \mathrm{yr}$, which is shorter than the SMBH growth time scale, and hence it satisfies a requirement for the adiabatic growth. The final SMBH mass is much larger than the initial mass that is probably similar to typical SMBH mass in normal galaxies $\left(10^{6-9} M_{\odot}\right)$, and hence another condition of adiabatic growth mentioned by [13] is satisfied.

Thus, it seems reasonable to suppose that the adiabatic spike growth occurred in the past, within $\sim \mathrm{kpc}$ of the cluster center where even the latest $\mathrm{X}$-ray satellites cannot resolve the density profile. Dominant energy production by annihilation occurs in the central core, where the core density $\rho_{c}$ is limited by annihilation itself over a typical cluster age, $t_{\mathrm{cl}} \equiv 10^{10} t_{10} \mathrm{yr}$, as $\rho_{c}\langle\sigma v\rangle t_{\mathrm{cl}} / m_{\chi} \sim 1$. Equating this core density and the spike density profile, $\rho=\rho_{0}\left(r / r_{s}\right)^{-\gamma_{s}}\left(r_{s} / r_{0}\right)^{-\gamma}$, I find the core radius $r_{c}=$ $0.17 M_{\bullet, 10}^{2 / 7} m_{2}^{-3 / 7}\langle\sigma v\rangle_{-26}^{3 / 7} t_{10}^{3 / 7}$ pc. (It is much larger than the Schwarzschild radius of the SMBH, 0.95M •,10 $\mathrm{mpc}$.)
The annihilation luminosity within this core radius is given by

$$
\begin{aligned}
L_{\chi \chi} & =2 m_{\chi} c^{2}\langle\sigma v\rangle\left(\frac{\rho_{c}}{m_{\chi}}\right)^{2}\left(\frac{4 \pi}{3} r_{c}^{3}\right) \\
& =1.9 \times 10^{44} M_{\bullet, 10}^{6 / 7} m_{2}^{-2 / 7}\langle\sigma v\rangle_{-26}^{2 / 7} t_{10}^{-5 / 7} \mathrm{ergs} \mathrm{s}^{-1} .
\end{aligned}
$$

This is a rate within $r_{c}$, and adding integration at $r>r_{c}$ increases the rate by a factor of 2.8. The above equation suggests a modest time evolution of $L_{\chi \chi} \propto t^{-5 / 7}$, and the time average over the cluster age is increased by a factor of $7 / 2$. Then I finally obtain $L_{\chi \chi} \sim 10^{45} \mathrm{ergs} / \mathrm{s}$, which is very close to the number required to heat the cooling cluster cores. In contrast to other astrophysical heating sources, this process is stable with only mild time evolution once it is turned on, unless the spike is disrupted by violent events such as major mergers. When such events destroy the spike, the cooling core may also be destroyed, changing a CF cluster into the other category of non-CF ones.

It must be examined whether this energy production is efficiently converted to the heat of intracluster gas. The annihilation products are eventually converted to stable particles. I used the DarkSUSY package [16] to calculate the amount and energy spectrum of these annihilation yields and found that, with only weak dependence on SUSY parameters, about $1 / 4,1 / 6$, and $1 / 15$ of the total annihilation energy goes to continuum gamma rays, $e^{ \pm}$'s, and $p \bar{p}$ 's, respectively. The other energy goes to neutrinos, which are not useful for heating. The spectral energy distribution of particles per logarithmic interval, $\epsilon^{2} d N / d \epsilon$, peaks at about $0.05,0.05$, and 0.1 times $m_{\chi} c^{2}$, in the same order. Therefore, most of the annihilation energy will be carried away by particles of about $5 \mathrm{GeV}$, for example, for $m_{\chi} \sim 100 \mathrm{GeV}$.

First, I consider the fate of electrons and positrons. For simplicity, I assume that a fraction $f_{ \pm} \sim 1$ of the total annihilation energy is given to $e^{ \pm}$and all $e^{ \pm}$'s have the same energy of $\epsilon_{0} \equiv \epsilon_{ \pm} /(1 \mathrm{GeV}) \sim 1$. They are produced in a very dense environment, and their relativistic motion results in much higher pressure than the environment. Therefore, $e^{ \pm}$'s expand until their pressure becomes comparable with the intracluster pressure. Buoyancy may result in intermittent formation of bubbles of relativistic particles. The $e^{ \pm}$density in the bubbles can be written by external pressure, as $n_{ \pm} \sim P_{\text {ext }} / \epsilon_{ \pm} \sim 6.3 \times$ $10^{-7} P_{-9} \epsilon_{0}^{-1} \mathrm{~cm}^{-3}$, where $P_{-9} \equiv P_{\text {ext }}^{-} /\left(10^{-9} \mathrm{erg} \mathrm{cm}^{-3}\right) \sim$ 1. This value and gas density $n_{-1} \equiv n /\left(0.1 \mathrm{~cm}^{-3}\right) \sim 1$ are taken from observed values within $r \leqq 1-10 \mathrm{kpc}$ [11].

The heating of intracluster gas by cosmic-ray electrons produced by AGNs has been discussed in literature $[17,18]$, and similar treatments can be applied to estimate the energy loss time scale of $e^{ \pm}$'s, $\tau_{ \pm}$. The upper limit on $\tau_{ \pm}$is given by the ordinary Coulomb collisions with a background gas: $\tau_{ \pm, \mathrm{cc}}=5.1 \times 10^{8} n_{-1}^{-1} \epsilon_{0}$ yr. This should be compared with the radiative energy loss time scale by 
inverse-Compton scattering (ICS) of the cosmic microwave background (CMB) photons, $\tau_{ \pm \text {,ic }}=1.2 \times$ $10^{9} \epsilon_{0}^{-1} \mathrm{yr}, \quad$ and synchrotron radiation, $\tau_{ \pm, \text {sync }}=$ $\tau_{ \pm, \text {ic }}\left(B / B_{\mathrm{CMB}}\right)^{-2}$, where $B_{\mathrm{CMB}}=3.30 \mu \mathrm{G}$. I also found that stellar radiation energy density in $\mathrm{CD}$ galaxies is comparable to that of $\mathrm{CMB}$, estimating it by $U_{\mathrm{st}} \sim$ $L_{\mathrm{cD}} /\left(4 \pi r_{\mathrm{cD}}^{2} c\right)$, where $L_{\mathrm{cD}}$ and $r_{\mathrm{cD}}$ are typical observed stellar luminosity and spatial size, respectively [11]. Therefore ICS of stellar photons should also have a comparable effect. The bremsstrahlung loss time scale is $\tau_{ \pm, \text {br }} \sim 5.7 \times 10^{8} n_{-1}^{-1} \mathrm{yr}$, and the annihilation time scale of positrons is $\sim 1.0 \times 10^{10} n_{-1}^{-1} \epsilon_{0}$ yr. Comparing these time scales, it can be seen that a considerable part of $e^{ \pm}$ energy can be converted into thermal energy.

Furthermore, even more efficient energy loss is possible when collective effects of plasma, such as the relativistic two-stream instability, are important [18]. An injection of a huge amount of relativistic $e^{ \pm \text {'s within }}$ the small core radius $r_{c}$ would lead to a strong wind of these particles, making their momentum distribution strongly anisotropic, which is necessary for the collective effect. Using formulas given in these references, I found $\tau_{ \pm, \text {tsi }}=3.1 \times 10^{3}\left(n_{ \pm} / n\right)^{-2}\left(n / \mathrm{cm}^{-3}\right)^{-0.5} \epsilon_{0}^{2} \mathrm{~s}=$ $8.3 \times 10^{6} P_{-9}^{-2} n_{-1}^{1.5} \epsilon_{0}^{4} \mathrm{yr}$. Dependence on $\epsilon_{ \pm}$is large, and almost all energy could be quickly converted to thermal energy when $\epsilon_{ \pm} \lesssim 1 \mathrm{GeV}$.

The annihilation energy given to $p \bar{p}$ 's would also be partially converted into thermal energy by at least the rate of Coulomb collisions that is similar to $\tau_{ \pm, \mathrm{ccc}}$. Some fraction of energy may be lost by inelastic interaction with ambient thermal protons, with a time scale of $\tau_{\mathrm{pp}} \sim$ $\left(n \sigma_{\mathrm{pp}} c\right)^{-1}=3.3 \times 10^{8} n_{-1}^{-1} \mathrm{yr}$, and secondary $e^{ \pm}$'s produced by this interaction would again be used for heating. It is likely that primary annihilation gamma rays do not contribute much to heat the cooling gas, because the optical depth to Compton scattering is $\sim 10^{-3} \ll 1$. However, if there are dense clouds having large optical depth around the annihilation core, the Compton heating may also have a considerable effect, as considered for heating by AGNs (e.g., [19]).

What is the preferred value of the neutralino mass in this context? The peak of $\epsilon^{2} d N / d \epsilon$ should be less than $\sim$ a few $\mathrm{GeV}$; otherwise the energy loss time scale for heating $\left(\tau_{ \pm, \mathrm{cc}}\right.$ or $\left.\tau_{ \pm, \mathrm{tsi}}\right)$ becomes longer than that for radiative loss, leading to inefficient heating. Combined with the relation $L_{\chi \chi} \propto m_{\chi}^{-2 / 7}$, the neutralino mass should be $\leqslant 100 \mathrm{GeV}$ for the proposed process to efficiently work.

Now I discuss the observability of any signature of the neutralino annihilation. Typically about $\sim 30$ continuum gamma rays are produced at $\epsilon_{\gamma}>100 \mathrm{MeV}$ per annihilation [16], and expected gamma-ray flux becomes $F_{\gamma}(>100 \mathrm{MeV}) \sim 7 \times 10^{-8} L_{45} m_{2}^{-1} d_{2}^{-2} \mathrm{~cm}^{-2} \mathrm{~s}^{-1}$, where $L_{45} \equiv L_{\chi \chi} /\left(10^{45} \mathrm{ergs} \mathrm{s}^{-1}\right)$ and $d_{2}=d /(100 \mathrm{Mpc})$ is a typical distance to nearby rich clusters. This is just almost the same as the EGRET sensitivity limit, and hence the prediction is marginally consistent with no reported gamma-ray detection from nearby galaxy clusters [20]. In fact, there are many positional coincidences between known galaxy clusters and unidentified EGRET sources, and the detection from clusters has not yet been claimed because of low statistical significance. The next generation gamma-ray satellite, GLAST will very likely detect the continuum gamma-ray flux as steady and point sources at cluster centers.

Even if the continuum gamma rays may be discriminated from other astrophysical sources by spectrum, variability, and/or extension, conclusive evidence would come from line gamma rays. Line flux (in $\mathrm{cm}^{-2} \mathrm{~s}^{-1}$ ) is expected to be smaller than the continuum flux by a factor of about $15 \times 10^{3}$, where the former factor $(30 / 2)$ comes from the ratio of the photon number produced per annihilation and the latter comes from the branching ratio into $\chi \chi \rightarrow \gamma \gamma$ or $Z \gamma$ modes [6]. Following the line sensitivity estimate given in this reference, I found that the line flux expected at $\lesssim 100 \mathrm{GeV}$ may produce several photons from a cluster center for a five-year operation of GLAST, compared with a negligibly small background rate of $\sim 10^{-3}$ events within angular and energy resolution. Co-added analysis of many CF clusters would even increase the sensitivity. Future air Cerenkov telescopes may have even better sensitivity for the line flux, but the threshold energy must be lower than $\sim 100 \mathrm{GeV}$ since we expect $m_{\chi} \lesssim 100 \mathrm{GeV}$.

Since the flux enhancement by the density spike is so drastic, the best target to search neutralino annihilation may be nearby CF clusters, rather than the GC or nearby galaxies. On the other hand, $L_{\chi \chi}$ without the density spike $\left(\sim 10^{40} \mathrm{ergs} / \mathrm{s}\right)$ is below the sensitivity limit even for the next generation gamma-ray telescopes.

Equally intriguing is the detectability of synchrotron radiation in the radio bands, by $e^{ \pm}$pairs produced by annihilation. It is known that central $\mathrm{cD}$ galaxies in $\mathrm{CF}$ clusters have a higher probability of becoming radio sources than those in non-CF clusters, and there seems to be a correlation between radio power and CF strength [21], suggesting that the annihilation $e^{ \pm}$may be responsible for radio emission. Typical radio luminosity from CF cluster cores is $\sim 10^{41-42} \mathrm{ergs} / \mathrm{s}$ [21], which is about $10^{3}$ times lower than the cluster $\mathrm{x}$-ray luminosity or $L_{\chi \chi}$. This factor can be explained by a few effects as follows. The magnetic field strength is poorly known, which may be weakened by relativistic bubble formation. Hard $\mathrm{x}$-ray tails observed in some cluster spectra are popularly interpreted as ICS of CMB photons by cosmic-ray electrons, and their luminosity is a few hundred times larger than diffuse radio halo luminosity, indicating that synchrotron is an inefficient process compared with ICS [22]. The energy loss by heating can be much more efficient than radiative loss by the collective effect, and then the total radio flux will be further reduced. Characteristic 
synchrotron frequency is $\nu_{\text {sync }} \sim 0.011 \epsilon_{0}^{2}(B / \mu \mathrm{G}) \mathrm{GHz}$. Since the injection of relativistic $e^{ \pm}$occurs in a relatively narrow energy range around $\sim 0.05 m_{\chi} c^{2}$ compared with the broad power-law spectrum of cosmic-ray electrons, most of the radio emission may occur at $\leq \mathrm{GHz}$, which is out of typical observing frequencies.

Here I give a size estimate of the radio emitting region. It is expected that the size of the $e^{ \pm}$bubble, $r_{b}$, is determined by the pressure balance and the lifetime of these particles, as $r_{b}=\left(3 \tau_{ \pm} \dot{N}_{ \pm} / 4 \pi n_{ \pm}\right)^{1 / 3}$, where $\dot{N}_{ \pm}=$ $f_{ \pm} L_{\chi \chi} / \epsilon_{ \pm}$is the $e^{ \pm}$production rate. Using $\tau_{ \pm, \text {tsi }}$ for the lifetime of $e^{ \pm}$, s, I found $r_{b}=13 n_{-1}^{1 / 2} \epsilon_{0}^{4 / 3} P_{-9}^{-1} f_{ \pm}^{1 / 3} L_{45}^{1 / 3} \mathrm{kpc}$. Typical extension of the radio emission associated with cD galaxies is $\sim 5-10 \mathrm{kpc}$ [21]. The large scale radio halos ( $\gtrsim \mathrm{Mpc}$ ) are found only in non-CF clusters like Coma, but CF clusters often have less extended "minihalos" ( $\leqslant 100 \mathrm{kpc})$ in which a strong radio cD galaxy is centered [23]. It may be speculated that the $e^{ \pm}$bubble corresponds to the central strong radio source, while radio minihalos are made by leaking $e^{ \pm}$from the bubble, perhaps by diffusion. The morphology of such radio galaxies and minihalos in CF clusters is poorly collimated and more spherical, compared with clear bipolar jetlike structures generally found in radio galaxies [21]. Such a trend may be difficult to explain if CF is suppressed by AGN jets, while it is naturally understood if the steady, isotropic energy production by neutralino annihilation is responsible. If this interpretation is true, it means that we have already observed, though indirectly, the dark matter for many tens of years.

Annihilation $e^{ \pm}$pairs would also produce a similar luminosity to synchrotron radiation in the $\mathrm{x}$-ray and $\mathrm{MeV}$ bands by ICS of CMB and stellar photons, respectively, but they are difficult to detect because of the strong thermal x-ray emission and difficulty of $\mathrm{MeV}$ observations.

The simple estimate of $L_{\chi \chi}$ without the density spike, $\sim 10^{40} \mathrm{ergs} / \mathrm{s}$, is comparable to the power of diffuse radio halos in merging clusters, and hence neutralinos may be the origin, as pointed out by Ref. [8]. A necessary condition for this is that the synchrotron must be the dominant energy loss process. However, as argued above (and in Refs. [22]), observed hard x-ray tails in cluster spectra indicate that the synchrotron is a rather inefficient process, and the diffuse synchrotron halo by annihilation $e^{ \pm}$'s is likely still under the current detection limit.

It is expected that the stars in the $\mathrm{cD}$ galaxies are also affected by the adiabatic growth, and it might be interesting to seek any signature in the central luminosity density profile of $\mathrm{cD}$ galaxies by high resolution optical observations. In fact, adiabatic growth by SMBHs is one of the proposed explanations of cusps seen in the central surface brightness profile of elliptical galaxies [24]. However, there is no reason to believe that the stellar density profile should be the same as that of dark matter, and large elliptical galaxies generally have flat stellar density cores [24]. A stellar density spike should be weak and may not be detectable, if it is formed from such a flat density core.

This work has been partially supported by a Grant-inAid for the COE program for physics at Kyoto University.

[1] M. Donahue and G. M. Voit, astro-ph/0308006, and references therein.

[2] J. N. Bregman and L. P. David, Astrophys. J. 326, 639 (1998); N. Zakamska and R. Narayan, Astrophys. J. 582, 162 (2003).

[3] F. Brighenti and W. G. Mathews, Astrophys. J. 573, 542 (2002), and references therein.

[4] A. Fabian, L. M. Voigt, and R. G. Morris, Mon. Not. R. Astron. Soc. 335, L71 (2002).

[5] G. Jungman, M. Kamionkowski, and K. Griest, Phys. Rep. 267, 195 (1996).

[6] L. Bergström, P. Ullio, and J. H. Buckley, Astropart. Phys. 9, 137 (1998).

[7] B. Qin and X. P. Wu, Phys. Rev. Lett. 87, 061301 (2001).

[8] S. Colafrancesco and B. Mele, Astrophys. J. 562, 24 (2001).

[9] D. A. Buote, Astrophys. J. 553, L15 (2001).

[10] J. F. Navarro, C. S. Frenk, and S. D. M. White, Astrophys. J. 490, 493 (1997); B. Moore, T. Quinn, F. Governato, J. Stadel, and G. Lake, Mon. Not. R. Astron. Soc. 310, 1147 (1999).

[11] A. D. Lewis, D. A. Buote, and J. T. Stocke, Astrophys. J. 586, 135 (2003).

[12] P. Gondolo and J. Silk, Phys. Rev. Lett. 83, 1719 (1999).

[13] P. Ullio, H. S. Zhao, and M. Kamionkowski, Phys. Rev. D 64, 043504 (2001).

[14] D. Merritt, M. Milosavljević, L. Verde, and R. Jimenez, Phys. Rev. Lett. 88, 191301 (2002).

[15] C. Jones and W. Forman, Astrophys. J. 276, 38 (1984).

[16] P. Gondolo, J. Edsjö, P. Ullio, L. Bergström, M. Schelke, and E. A. Baltz, astro-ph/0211238.

[17] S. M. Lea and G. D. Holman, Astrophys. J. 222, 29 (1978).

[18] J. S. Scott, G. D. Holman, J. A. Ionson, and K. Papadopoulos, Astrophys. J. 239, 769 (1980); R. Rosner and W. H. Tucker, Astrophys. J. 267, 547 (1983).

[19] L. Ciotti and J. P. Ostriker, Astrophys. J. 551, 131 (2001).

[20] O. Reimer, M. Pohl, P. Sreekumar, and J. R. Mattox, Astrophys. J. 588, 155 (2003).

[21] J. O. Burns, Astron. J. 99, 14 (1990); R. Ball, J. O. Burns, and C. Loken, Astron. J. 105, 53 (1993); J. Bagchi and V. K. Kapahi, J. Astrophys. Astron. 15, 275 (1994).

[22] R. Fusco-Femiano et al., Astrophys. J. 513, L21 (1999); V. Petrosian, Astrophys. J. 557, 560 (2001); L. Rudnick and K. M. Blundell, Astrophys. J. 588, 143 (2003).

[23] J. O. Burns, M. E. Sulkanen, G. R. Gisler, and R. A. Perley, Astrophys. J. 388, L49 (1992).

[24] R. P. van der Marel, Astrophys. J. 117, 744 (1999). 